\title{
Research on the Inheritance and Development of Linear Skills in Guzheng Performance
}

\author{
Le Jingqi \\ Traditional Instruments Department, Sichuan Conservatory of Music, Chengdu, China
}

Email address:

Le_jingqi@163.com

\section{To cite this article:}

Le Jingqi. Research on the Inheritance and Development of Linear Skills in Guzheng Performance. International Journal of Literature and Arts. Vol. 9, No. 4, 2021, pp. 173-176. doi: 10.11648/j.ijla.20210904.15

Received: July 8, 2021; Accepted: July 19, 2021; Published: July 24, 2021

\begin{abstract}
As a member of plucked stringed instruments, Guzheng, with its granular sound characteristics, constitutes the melody and lines with a strong Chinese national style. The Guzheng playing technique is made up of 4 point like sound in the early spring and autumn period, such as "Tuo", "Muo", "Gou", and "Da" (transliterate), and later developed and innovated linear music skills such as "Tuopi", "Yaozhi", "Lunzhi” (transliterate), etc. Guzheng art has a long history, and its playing techniques are constantly innovating. These innovations in linear techniques give the Guzheng, which is a traditional plucked instrument, the opportunity and possibility to better express linear music. In order to explore the meaning and effect of the linear technique of Guzheng performance on the art of Guzheng performance, this study systematically analyzes the development status, performance effects and training methods of the three Guzheng playing techniques, Tuopi, Yaozhi, Lunzhi. This study further analyzes the technical characteristics of the three techniques of Tuopi, Yaozhi, Lunzhi in guzheng playing, and draws conclusions about the importance of "linear" art in the development of Guzheng. It not only provides references for the performers in the acquisition of the three skills systematically, accurately and scientifically, but also helps the performers to improve their performance levels.
\end{abstract}

Keywords: Guzheng Performance, Linear Music, Playing Technique, Tuopi, Yaozhi, Lunzhi

\section{Introduction}

Guzheng is a kind of national musical instrument in China, it is a plucked stringed instrument [1]. The pronunciation form of "point" is the basic sound form of Guzheng. The series of performance techniques formed by this way of pronunciation are called "point" technique in this paper. The "line" in the guzheng pronunciation form is composed of continuous and dense "point", forming a "line" pronunciation form. The performance technique formed from this is called "linear" technique in this paper. In the early Guzheng playing methods, more point techniques were used, and it was more difficult to obtain the desired effect when playing linear music [2]. Therefore, the linear music performance techniques based on "Tuopi", "Yaozhi", "Lunzhi" (transliterate) allow the Guzheng to perform linear melody while playing pointed music. Music is rhythmic and coherent, so the concept of "linear" can satisfy these properties well. The performer uses playing techniques to combine point and line sound like. While showing granular sound effects, Guzheng can also show the continuity and diversity of musical melody lines. It shows that in the course of the development of Chinese national music for more than two thousand years, the Guzheng playing technique has also been continuously developed and improved [3, 4]. A correct understanding of the linear techniques in Guzheng playing is of instructive significance for mastering the various styles of Guzheng, inheriting and developing the Guzheng techniques with Chinese characteristics and conforming to the national aesthetic psychology.

This paper analyzes the history, development process and technical characteristics of three techniques, such as Tuopi, Yaozhi, Lunzhi in the linear technique of Guzheng playing [5]. It provides reference for Guzheng players to master these three performance techniques in a more systematic, standardized and scientific manner, and helps improve the level of Guzheng performance. In addition, this paper also discusses the application of the three performance techniques in different musical repertoires, laying an important theoretical foundation for the cultivation and promotion of 
Guzheng performers' techniques.

\section{Types of Linear Techniques in Guzheng Performance}

\subsection{Tuopi}

"Tuopi" is a representative technique in the traditional genre of Guzheng [6]. Among the nine traditional genres of Guzheng [7,8], the northern genre of Guzheng uses the Tuopi technique more. The basic method of Tuopi is to combine the right-hand playing technique "Tuo" (transliterate) ( $ا$ ) and "Pi" (transliterate) $(\neg)$ ) [9]. When playing Tuo, the small joints of the big finger support the Guzheng board and stick to the string to play. Pi belongs to the reverse fingering of the support using the downward force of the Tuo to lift the big or small joints of the big finger upwards. This fingering is often used interchangeably with the Tuo. There are differences in the application of Tuopi in different Guzheng genres, such as large joint Tuopi, small joint Tuopi and hanging wrist Tuopi (that is, use the index finger to pinch the fingertip of the big finger, and use the power of the wrist to Tuopi) [2]. Different types of Tuopi are played differently in different genres of Guzheng.

\subsection{Yaozhi}

"Yaozhi" takes the wrist as the axis and uses a finger or multiple fingers on one or more strings, and repeats rapid plucking back and forth many times. Quickly flick the sound back and forth, and connect the points of each sound in a dense frequency to maintain the full duration of the sound and the continuity of the music, thereby prolonging the duration of the note. The Yaozhi of the Zhejiang Guzheng genres is similar to the current Yaozhi, and its techniques are developed on the basis of fully inheriting the Zhejiang Guzheng genres Yaozhi. Shaking fingers has always played an important role in the long-term development of Guzheng. Through the application and practical exploration in different types of music, it has developed a variety of types, such as big finger Yaozhi, index finger Yaozhi, multi-finger Yaozhi, left hand Yaozhi, and two hands Yaozhi. In addition, "big finger Yaozhi" can be subdivided into "wrist Yaozhi”, "sweep Yaozhi” and so on. The emergence of Yaozhi reflects the requirements for linear techniques in the development of Guzheng playing techniques.

\subsection{Lunzhi}

"Lunzhi" means that the big finger, index finger, middle finger, and ring finger play the same thread in sequence in the same direction or opposite direction to perform continuous rounds. When the sound duration and volume of the four fingers are close to the same as the timbre, after reaching a certain speed, a coherent melody will appear, which is aurally similar to finger shaking. Since the four fingers have strong independence, the effect of this technique in actual performance is more flexible, and the timbre changes are more abundant. Lunzhi is a new playing technique developed by Mr. Zhongshan Wang, who has fully borrowed from the Lunzhi playing technique of the Pipa and combined with the characteristics of the Guzheng itself [10]. In the process of continuous development, various types such as "play Lunzhi", "three-finger Lunzhi" and "left-hand Lunzhi" have been appeared, which are more suitable for the performance of modern music.

\subsection{Comparison of the Three Techniques}

The main difference of the three linear performance techniques of Tuopi, Yaozhi, Lunzhi is the different ways of exerting force, the different timbre, and the specific music used. For example, most of the traditional Guzheng music has a slower rhythm and does not have such high technical requirements. When the Lunzhi technique does not appear, the linear melody is often used Tuopi. Most modern Zheng music has high technical requirements, so various types of Yaozhi and Lunzhi are more suitable for modern works.

The author found through consulting related literature that many literatures classify the technique of Tuopi into the category of Yaozhi. However, Tuopi and Yaozhi are not the same in terms of hand shape, force method and timbre. The chopping technique requires a lot of practice to produce a granular melody, while shaking fingers can easily show a fine and coherent melody. It can be seen from the above that Yaozhi is an innovation and development on the basis of Tuopi. However, in the actual performance of Yaozhi, there will be problems such as adjusting the angle when shaking the fingers in different zones, so the Lunzhi technique appears. Lunzhi adjusts tones in different zones more freely. It can be seen that the relationship between the three is a process of continuous inheritance, with close connections, and also shows the process of continuous reform, continuous innovation, and continuous development of the Guzheng linear technique [11, 12].

\section{The History and Development of Guzheng Linear Technique}

\subsection{The History of Guzheng Linear Technique}

In the history of the Guzheng, the linear technique of the Guzheng can be inferred as early as the Tang Dynasty's music collection RenZhiYaoLu (transliterate). This collection of scores was compiled by the Japanese Moronaga Fujiwara in 1171. It provides us with valuable information for studying the shape, score, performance style and technique of the Guzheng music in the Tang Dynasty. The ZhengAnPuFa Method (transliterate) in the first volume of RenZhiYaoLu (transliterate) explains the name of Tang Zheng's string and the left and right hand playing techniques. The right hand on the right represents the right fingering, the fingering of the big finger of the spectrum character (the Tuo in the current Guzheng playing technique, fingering symbol: L), and the Fanzhua (transliterate) fingering under the big finger is playing in the opposite direction of the big finger (the split in the current guzheng playing technique, fingering symbol: $\urcorner$ ), the long duration is "Yao". The performance technique of the big finger 
in the score of RenZhiYaoLu (transliterate) includes Tuo and Pi. From this we can infer the possibility of its continuous use in actual performance. If the Tang people used the big finger and Fanzhua (transliterate) to extend the sound value and click the sound to form a line, then there may have been a big finger "Tuopiyaozhi" (transliterate) technique in the Tang Dynasty.

XianSuoBeiKao (transliterate) is a thirteen instrumental score composed mainly of stringed instruments by Rong Zhai in the early Qing Dynasty. The scores and fingerings of the 13 instrumental music in the collection are all complete, so it has a very important historical position and practical value. Rong Zhai clearly indicated the fingering introduction of various string instruments such as Sanxian, Pipa, Zheng, etc. in the Fingering Collection of volume 1 of XianSuoBeiKao (transliterate) [13]. Among them, there are 16 right-hand playing techniques of Guzheng. The description of "|" refers to the technique of continuous chopping with the big finger, which is similar to the technique of Yaozhi today. In the thirteen pieces of XianSuoBeiKao, there are many different types of "|" techniques, such as short and long swings with different durations, finger swings with glide, and double swings.

In XianSuoBeiKao and WuMingMa (transliterate), the Guzheng double-shaking technique has appeared many times. The "I" in XianSuoBeiKao (transliterate) is similar to the "double-shaking" technique. A lot of linear techniques such as chopping, finger shaking, and double shaking are used in the music of XianSuoBeiKao (transliterate). It can be seen that many people think that difficult finger shaking like "double shaking" is a fingering method invented only in modern times. As everyone knows, it was widely used in WuMingMa (transliterate) and other songs a hundred years ago, showing that the Guzheng playing technique had a very high level of development a hundred years ago [13].

\subsection{The Development of Guzheng Linear Technique}

It can be seen from the above that the Tuo and Pi techniques of Guzheng have appeared in the Tang Dynasty, and the Yaozhi and "Double Yaozhi" techniques have appeared in the Qing Dynasty. For example, the representative of the Henan Guzheng genre is the large joint Tuopi, which is known for its full sound quality, usually with a clear and powerful sound head, and its performance is solid, bright, and sonorous. The representative of the Shandong Guzheng genre is the small joint Tuopi, which has a fine sound and a small graininess, and has a strong expressive force without the large joints that force the roots. The representative hanging wrist Tuopi in the repertoire of the Shaanxi Guzheng genre. Because it uses a hanging wrist, the accuracy is low, so it often splits a piece of sound instead of a single sound. The hanging wrist rest is more expressive and infectious, and the rendering of music becomes more intense.

In the 1970s and 1980s, a large number of composers inherited traditional Chinese composition techniques, and borrowed Western composition techniques to create a large number of modern Guzheng works. These works not only promoted the development of Guzheng music, but also promoted the progress of Guzheng playing techniques. In the long historical development process, there are many kinds of Yaozhi. For example, Saoyao's sound effects are quite imposing, applied to scenes of intense tension and majestic spirit, greatly enriching Yaozhi's expressiveness. Yaozhi techniques were played with the right hand before the 1990s, and left-handed Yaozhi began to appear after the 1990s. The technique of Yaozhi with both hands is also fully used in the music "Yunling Music and Painting". The use of the left hand Yaozhi is a breakthrough in the traditional Guzheng playing technique, which not only enriches the Guzheng playing technique, but also creates a new development situation for Yaozhi.

The modern Guzheng music technique also draws lessons from other plucked instruments' playing techniques, which enriches the form of expression of the zheng. Lunzhi is a playing technique borrowed and developed from the playing techniques of pipa and classical guitar. The Lunzhi technique has been widely used since the 1990s. After it first appeared in the work "Dance of the Yi People" adapted by Mr. Zhongshan Wang, it has received enthusiastic response, and new techniques such as "Left Hand Lunzhi" have been derived [14]. Lunzhi effectively solves the timbre problem when playing the Guzheng long tone, making the timbre more mellow and full. The long-lasting endurance and easy-to-adjust frequency enable Guzheng to play complex, multi-modal and difficult modern works at will. The Lunzhi technique expands the artistry and performance space of the Guzheng playing technique, and increases the expressiveness of the Guzheng music structure. Since the founding of the People's Republic of China, the Guzheng technique has undergone a transformation from an early single technique to a diversified technique. From the ancient Pituo and Yaozhi to the present multiple types of Pituo, Yaozhi and Lunzhi, this all reflects that the Guzheng linear technique inherits the traditional technique, dare to learn and borrow, and dare to explore and innovate. The production and application of these linear techniques not only broadened the Guzheng playing techniques, but also greatly enhanced the expressiveness of Guzheng playing.

\section{Conclusion}

National culture has shaped the Chinese nation's special way of thinking and aesthetic concepts, making musicians pursue neutrality, elegance, and tranquility in musical aesthetics. This has contributed to the music and instrumental music of the Chinese nation, and formed the instrumental aesthetics with linear beauty as the main feature [15]. Through the comparative study of the three linear techniques mentioned above, it is not difficult to see that the emergence of linear techniques such as Tuopi, Yaozhi and Lunzhi make the Guzheng playing technique not only have "point" like sound but also "lines" sound like [5]. Three linear techniques have an important position in each era of the development of Guzheng. This shows that linear art is of great value to the development of Guzheng. When we 
research, inherit and develop Guzheng playing techniques, we must not only inherit and retain traditional techniques, but also create new techniques that can adapt to modern Guzheng works. It is necessary to meet the characteristics of the development of modern Guzheng art, but also cannot replace or abandon traditional performance techniques at will. Only when we continue to explore and innovate and combine "point" with "lines" can we promote the development of Guzheng art.

In summary, the creation of Guzheng art not only needs to inherit the traditional advantages of the ancients, but also needs to face the current needs of reality, and continue to inherit and innovate the Guzheng art. Only in this way can the traditional Guzheng art radiate stronger vitality at present, and be able to maintain its charm forever in the art palace.

\section{References}

[1] Yang Qi. The Application of Minority Music in the Teaching of Guzheng in Higher Vocational Education]. Institute of Management Science and Industrial Engineering, 2019: 4.

[2] Peng Shanxia. Research on functions and techniques of special sound in Guzheng performance. International Conference on Education, Management and Computing Technology (ICEMCT 2015), (2015) 6-9.

[3] Rui Liu. A Combination of Chinese and Western Elements-Research of the Innovative Musical Language of Guzheng Works. Information Engineering Research Institute, USA: Information Engineering Research Institute, 2013: 5.

[4] Peng Shanxia. Research on Functions and Techniques of Special Sound in Guzheng Performance. Proceedings of 2015 2nd International Conference on Education, Management and Computing Technology (ICEMCT 2015), 2015: 4.

[5] Yao Shengjun. A brief talk on the development of Guzheng playing techniques-take the development of "Pi Tuo", "Yaozhi", "Lunzhi" as an example. Popular Literature and Art, 2010 (24): 7-8.
[6] Meng Yang. Investigation on the nine art genres of Guzheng in contemporary China. The Voice of the Yellow River. 2014, (18): 87-89.

[7] Xun Wang. The Cause of formation of the Guzheng genre from the perspective of regional culture. Chinese Music. 2009, (04): 147-152.

[8] Mingming Qu. The Development and Innovation of Chinese Guzheng Playing Techniques. Research Institute of Management Science and Industrial Engineering. Proceedings of 2017 4th International Conference on Literature, Linguistics and Arts (ICLLA 2017), 2017: 5.

[9] Huiying Liu. The application of the Guzheng playing technique "Tuo Pi" in Henan Zheng music and Shandong Zheng music. Art Criticism, 2017 (24): 71-72+116.

[10] Murong Lin. One sound, one world-Interpretation of the importance and technical difficulty of mid-long tone in Guzheng Music Performance. Chinese National Expo, 2021, 03 (05): 147-149, 168.

[11] Wang Xiaomei. Analysis of the Characteristics and Works of Traditional Guzheng Performance. International Information and Engineering Association. Proceedings of 2018 1st International Conference on Education, Art, Management and Social Sciences (EAMSS 2018), 2018: 5.

[12] Xiaomei Wang. A Discussion on the Application of Traditional Rhythm Techniques in Modern Guzheng Performance. International Journal of Intelligent Information and Management Science, 2019, 8 (2).

[13] Ye Sang. A Study on the Traditional Techniques and Historical Value of Guzheng in WuMingMa. Nanjing University of the Arts, 2014.

[14] Mengge Tan. Analyze the "dot", "line" and "face" expressed in the music of the "Lunzhi" playing technique of Guzheng. Artwork Jian, 2021 (09): 161-162.

[15] Hu Xianwen. Contemporary cultural characteristics of modern Chinese Guzheng playing techniques. The Voice of the Yellow River, 2019 (20): 56-59. 